\title{
Modelo de colaboração sistêmica: potencializando o compartilhamento do conhecimento no ecossistema das organizações
}

\section{Systemic collaboration model: boosting knowledge sharing in the organizational ecosystem}

\author{
Thais Andrade Colicchio ${ }^{1}$, Antônio Carlos Zambon ${ }^{2}$ \\ 1 Universidade Estadual de Campinas (UNICAMP), Campinas, São Paulo, Brasil. ORCID: https://orcid.org/0000-0002-2625-3597 \\ 2 Universidade Estadual de Campinas (UNICAMP), Campinas, São Paulo, Brasil. ORCID: https://orcid.org/0000-0003-3925-2769
}

Autor para correspondência/Mail to: Thais Andrade Colicchio, t263015@dac.unicamp.br

Recebido/Submitted: 13 de maio de 2021; Aceito/Approved: 08 de setembro de 2021

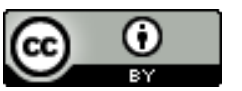

Copyright (C) 2021 Colicchio \& Zambon. Todo o conteúdo da Revista (incluindo-se instruções, política editorial e modelos) está sob uma licença Creative Commons
Atribuição 4.0 Internacional. Ao serem publicados por esta Revista, os artigos são de livre uso em ambientes educacionais, de pesquisa e não comerciais, com
atribuição de autoria obrigatória. Mais informações em http://revistas.ufpr.br/atoz/about/submissions\#copyrightNotice.

\begin{abstract}
Resumo
Introdução: esse short paper tem o propósito de contribuir com a compreensão das conexões entre Colaboração e Compartilhamento do Conhecimento, no âmbito da Gestão do Conhecimento (GC) nas organizações. Visa à evidenciar que a Colaboração, entendida como um sistema complexo, está composta por diversos níveis de interações entre agentes. Para compreender a leitura desse ecossistema, é necessário reconhecer os elementos que o compõe, buscando potencializar os impactos da Colaboração para as organizações. Método: a pesquisa é baseada nos dados coletados em revisão sistemática da literatura, e propõe uma leitura do conceito de Colaboração a partir dos Sistemas de Informação (SI) ao adotar uma abordagem de análise combinada dos resultados, mapeando conceitos associados ao Compartilhamento do Conhecimento, a partir de uma base qualificada por 270 artigos com foco em Colaboração. Na primeira etapa, explorou-se a densidade das conexões entre os conceitos de Colaboração e Compartilhamento do Conhecimento. Resultados: como sequência, desenhou-se em um modelo conceitual de Colaboração, que foi baseado nos apontamentos da análise inicial, representado por um framework que permite a leitura do sistema social, como um ecossistema de Colaboração, descrevendo a "Colaboração Sistêmica". Os resultados posicionam essa pesquisa na linha contemporânea, a partir de uma macro leitura de sistemas complexos, e complementam, assim, as pesquisas já existentes em SI e GC. Conclusão: nesta abordagem aplicada, a leitura estratégica da Colaboração Sistêmica incentiva a colaboração, e aponta potenciais influências no ecossistema da organização e na sustentabilidade das relações dos agentes.
\end{abstract}

Palavras-chave: Ecossistemas; Sustentabilidade; Sistemas Complexos; Gestão do Conhecimento; Sistemas de Informação.

\begin{abstract}
Introduction: the purpose of this short paper is to contribute io expanding the investigation of this research on the connections between Collaboration and Knowledge Sharing, based on the Knowledge Management (KM) of organizations. That means providing evidence that Collaboration, designed as a complex system, is built on different levels of agent interactions. In order to better understand this ecosystem, it is necessary to recognize the elements that inhabit this environment and then seeking to boost the impacts of Collaboration on organizations. Method: based on data collected at the systematic literature review that offers a point of view about collaboration from Information Systems (IS) perspective and implementing a combined analysis of the results, mapping concepts associated with knowledge sharing, in 270 articles reviewed. The goal of its first stage was to explore the connection's density between the Collaboration and Knowledge Sharing concepts. Results: as a consequence, the design of a conceptual collaboration model, based on the main findings of the previous analysis, represented here as a framework that is read as a social system, as a Collaboration Ecosystem, describing the "Systemic Collaboration". The results set this research in a contemporary context of complex systems overviews and connected it with IS and KM research. Conclusion: by offering an applied and strategic approach in reading the Collaboration as systemic, it both incentivizes the collaboration and lights its potential results for the ecosystem of the organization, building on sustainable relations between the agents.
\end{abstract}

Keywords: Ecosystem; Sustainability; Complex Systems; Knowledge Management; Information Systems.

\section{INTRODUÇÃO}

Ao abordar um tema multidisciplinar como o da Colaboração, este trabalho se pauta na compreensão de sistemas complexos. Segundo Nobre (2019), esse é o vetor que descreve o aumento da complexidade e também exige diferentes níveis de colaboração, que sejam capazes de gerar "enriquecimento" compartilhado no sistema. O autor aponta que ocorrerá um crescimento exponencial nessa catálise de conhecimento, a partir dos processos de consciência coletiva, onde impera a colaboração, afirmando que a complexidade só pode existir por meio dela. Aponta que a complexidade é a base e essência para nossa existência, e, sem ela, não existiria um sistema autorregulado (Nobre, 2019).

Baseados na tríade Pessoas, Processos e Tecnologia (PPT), no âmbito da abordagem em Gestão do Conhecimento (GC), considera-se como desafio, a busca por uma sinergia interdisciplinar, necessária à abordagem das complexas questões de gestão organizacional (Neves, Varvakis, \& Fialho, 2018).

Observam-se as pessoas como agentes protagonistas de seu sistema social e por isso torna-se necessário reconhecer a presença da Tecnologia Social (TS), a partir de uma via dupla de influência entre o grupo social e os impactos 
dos artefatos tecnológicos nas relações sociais que se desdobram ao seu redor (Dagnino, 2010). Essa mútua influência atua como um processo de construção de inteligência coletiva pelas trocas entre agentes do sistema. Nesta leitura, a TS é considerada um vetor (movimento bottom-up), não linear, que produz crescentes resultados desde a coleta de dados, uso de informação, compartilhamento de conhecimentos e construção de inteligência, como proposto pela hierarquia Data-Information-Knowledge-Wisdom (DIKW), e segundo os níveis hierárquicos da informação (Moresi, 2000).

Esta pesquisa propõe a modelagem em sistemas complexos (Systems Design Principles), e visa à compreensão holística do sistema de compartilhamento do conhecimento. Tem como objetivo descrever o processo de construção da inteligência coletiva, onde a colaboração, ao ser incentivada, pode potencializar os resultados da organização e o impacto positivo em todo o ecossistema. Busca-se assim, construir relações sustentáveis de sinergia entre os agentes do sistema. Ao modelar Colaboração Sistêmica focando nos impactos positivos, buscou-se nesta combinação com o Compartilhamento do Conhecimento apontar um propósito para a Colaboração.

Com esse propósito, foram identificadas 270 publicações relevantes, as quais foram analisadas a fim de propor os temas em quatro domínios: "Gestão de Empresas" - combinando o "Planejamento Estratégico" e "Inteligência Organizacional"; "Gestão do Conhecimento"- combinando a "Aprendizagem Cooperativa" com a GC para incentivar o uso do conhecimento dos agentes combinado em inteligência coletiva do sistema; "Sistemas de Informação"- e assim incentivar o mapeamento pelo Hub de Conhecimento. Por fim, uma leitura do sistema social do modelo da "Colaboração Sistêmica" tem como objetivo incentivar a construção de relações sustentáveis de longo prazo no "Ecossistema das Organizações", promovendo a escalabilidade da Colaboração, e consequentemente, a evolução de impactos positivos.

Este short paper foi organizado nas seguintes seções: a Introdução, aqui apresentada com o contexto da pesquisa e apresentação dos principais referenciais teóricos, seguido da apresentação dos procedimentos metodológicos desenvolvidos e os resultados apresentados. A seção de resultados traz, primeiramente em formato de figuras, as visualizações e análises que embasam a construção de um modelo teórico preliminar do Framework do Ambiente de Colaboração Sistêmica. Buscou-se apresentar o modelo preliminar e detalhar seu desenvolvimento, pautado pelos princípios do design de sistemas complexos e propondo sua leitura da dinâmica com analogias entre outros modelos teóricos para seu entendimento. Por fim, na seção de conclusão, foram apontadas as implicações teóricas e possíveis aplicações, além dos próximos desdobramentos esperados desta pesquisa.

\section{PROCEDIMENTOS METODOLÓGICOS E RESULTADOS}

O desenvolvimento do short paper de conceitualização do "Modelo de Colaboração Sistêmica" foi apoiado pelo Grupo de Pesquisa em Engenharia da Informação e Conhecimento (GEICon) da Faculdade de TecnologiaUnicamp, no macro tema "Gestão de Pessoas". O conteúdo aqui apresentado foi extraído dos dados coletados e tratados segundo metodologia declarada:

a) Etapa I - Revisão Sistemática (Kitchenham \& Charters, 2007) - condução sob protocolo do Método PICOC (Software: Parsif.al), nas bases Scopus, Web of Science e Periódico Capes: - "Business" OR "Company" OR *Organization*) AND ("Collaboration") AND ("Team Work" OR "Group Work" OR "Collaboration Environment" OR "Knowledge Sharing" OR "Shared Mental Model") AND ("Model" OR "Analysis Model" OR "Indicators" OR "System") - outcomes; - a pesquisa resultou em uma base qualificada de 270 publicações revisadas e aceitas.

b) Etapa II - Análise Qualitativa por meio do Software IRAMUTEQ (Interface de R - pour les Analyses Multidimensionnelles de Textes et de Questionnaires): - a partir do vocabulário do corpus, foi possível obter os gráficos de Nuvem de Palavras e Classificação Hierárquica Descendente (CHD). A CHD visa à classificar e apresentar os vocabulários semelhantes entre si por meio de diversos testes qui-quadrado, particionando o corpus em classes (Camargo \& Justo, 2007), sendo que o software organiza a análise de dados para representar as relações entre as classes.

Os resultados transcritos neste short paper visam à: 1. Tratar, organizar e compartilhar os dados de pesquisa; 2. Propor uma construção colaborativa para o grupo de pesquisa sobre este modelo, que representa o sistema em desenvolvimento; 3. Publicar o modelo preliminar e abrir espaço para contribuições no contexto do ecossistema das organizações.

Espera-se, assim, embasar a leitura do framework proposto, desenhado no software Insight Maker, como "Modelo Conceitual da Colaboração Sistêmica", baseado na modelagem soft de sistemas (?).

Resultados Preliminares: ao contextualizar a colaboração como um tema multidisciplinar, é importante apontar as diferentes áreas de estudo em questão. Essas áreas foram identificadas a partir dos journals ou eventos das publicações coletadas por essa pesquisa, como descrito na nuvem de palavras da Figura 1. 


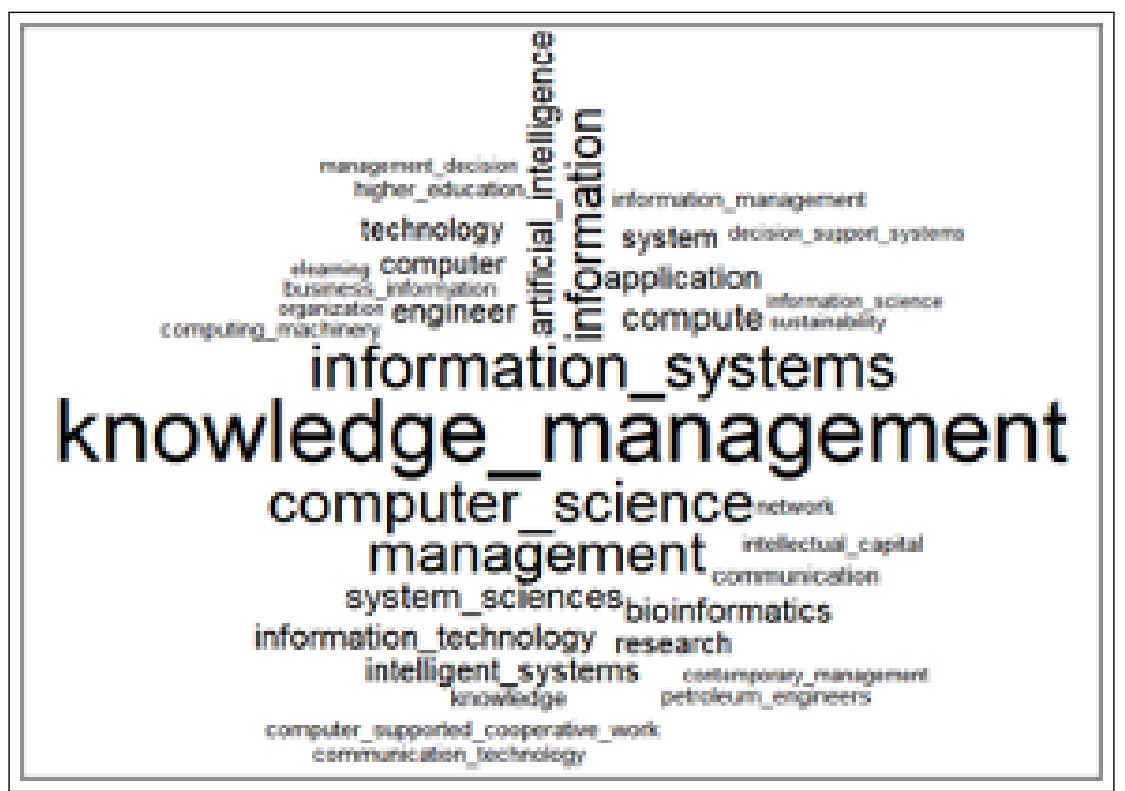

Figura 1. Áreas de Estudo. Fonte: Os autores (2021).

Observa-se na Figura 1 que os trabalhos coletados foram direcionados às bases de Gestão do Conhecimento, Sistemas e Sistemas de Informação, Ciência da Computação e Gestão de Empresas (Management/Business). Destaca-se também a presença de revistas e eventos especializados em Colaboração com três publicações em "Collaboration Technologies and Systems" e outras oito especializadas a exemplo da "Managing knowledge for global and collaborative innovations". Outro ponto em destaque é o papel das Tecnologias da Informação e Comunicação (TICs) e da ciência da computação no desenvolvimento de ambientes virtuais e ferramentas digitais de colaboração, exemplo da área "Collaborative-Computing".

Para pautar a continuidade das análises, a Figura 2 foi construída com a clusterização em classes de palavras, a partir das correlações baseadas nos títulos e abstracts das 270 publicações da base coletada. A Figura 2 representa a correlação central entre "Conhecimento" x "Colaboração", sendo que essa centralidade aponta que estas são as palavras em comum entre as três classes. 


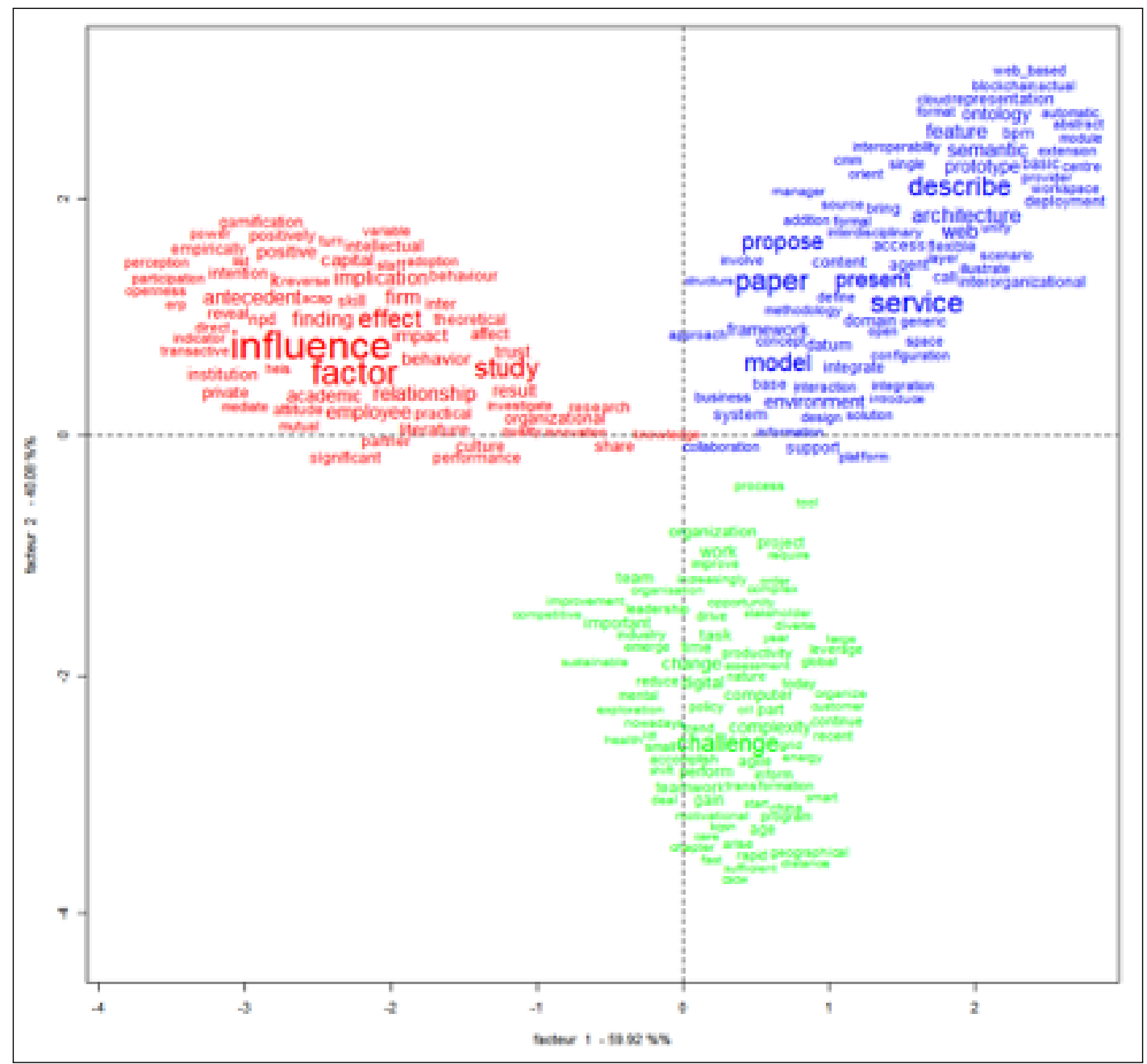

Figura 2. Clusterização em Classes de Palavras. Fonte: Os autores (2021).

$\mathrm{Na}$ figura, estão representadas as 3 classes identificadas no corpus de texto estudado:

CLASSE 1 (Vermelha 31,2\%) // CLASSE 2 (Verde 35,6\%) // CLASSE 3 (Azul 33,2\%), que tiveram um balanço de representatividade entre aprox. 31-35\%. Ao observar a Figura 2, pode-se ver a proximidade entre colaboração com o compartilhamento do conhecimento, conforme evidenciado nas três classes diferenciadas pelas cores. Além disso, a figura permite observar a proximidade dos conceitos sendo que o elo central observado "Conhecimento Colaboração"; também seguido em cada classe por "Compartilhar > Conhecimento" em vermelho; "Business Sistema - Informação - Suporte - Plataforma > Colaboração" em azul; e "Processo - Ferramenta" na terceira classe em verde. Ainda neste eixo central, gravitando ao centro próximo aos conceitos "Conhecimento - Colaboração" destacam-se os termos: "Ambiente", "Organizacional", "Relacionamentos", "Projeto" e "Trabalho". Outra leitura proposta é a análise de peso dos conceitos por sua frequência em cada classe, representados pelo tamanho de cada palavra na Figura 2. Assim, na classe verde se destaca a presença do "Desafio" > seguido por "Mudança" e "Trabalho"; "Time", "Projeto" e "Organização". Na classe vermelha de "Influência" > "Fator", "Efeito"; "Implicação", "Estudo" > associados ao "Conhecimento". No contexto da classe azul estão "Descrever", "Proposito", "Paper", "Serviço", "Modelo" > associados a "Colaboração".

As combinações propostas acima se alinham a um dos propósitos para a Colaboração (Wood \& Gray, 1991), o de construir a Inteligência Coletiva no Ecossistema das Organizações, a qual é necessária para produzir impactos positivos no sistema como um todo, frente aos desafios e complexidade em destaque. Avançando a partir desta leitura inicial para a Colaboração, buscou-se também responder ao desafio de identificar a multidisciplinaridade no estudo da Gestão do Conhecimento (GC). Ao propor um modelo, pretende-se contribuir para reforçar a percepção de que, para atingir os resultados esperados com a GC, é necessário considerar os fatores interno e externo às organizações (Neves et al., 2018). Assim, com a modelagem de um sistema social, buscou-se a sinergia entre diferentes áreas do conhecimento. Essa leitura da Tecnologia Social representa construir uma comunidade de pesquisa em um lugar/linguagem comum da Ciência e Tecnologia e Ensino (Dagnino, 2014).

Para introduzir o modelo preliminar que representa as condições do Ecossistema de Colaboração da organização, buscou-se apontar as sinergias entre os agentes e seus objetivos em comum como relações win-win-win (de sinergia e impacto positivo). Com o objetivo de descrever as sinergias neste sistema, assim, buscando complementar os 
estudos em Colaboração propondo um modelo de sistema complexo.

Ainda que os "Fatores e Influência", "Modelos e Ambientes" e "Desafios e Mudanças" sejam conceitos presentes nos documentos levantados, esses não são os conceitos mais próximos ou abordados em conjunto. Apontamos, assim, uma lacuna e uma oportunidade de desenvolvimento de um novo modelo que descreva o ambiente de colaboração nas organizações, pautado no conceito de um Sistema Sociotécnico Complexo (Complex Sociotechnical Systems), conforme proposto por Norman e Stappers (2015), como uma metodologia de design de sistemas centrado nas pessoas.

Neste Framework, desenhou-se uma abordagem para a Colaboração Sistêmica (Figura 3) como um sistema complexo, cujas características são apontadas (Systems Design Principles): Sistema Aberto, Network Descentralizado e Ciclo Vivo de Desenvolvimento, descritos pelas abordagens Holísticas e Relacional. Por isso, é necessário reconhecer que as diferentes camadas das relações entre os agentes podem produzir com a intensidade das relações, neste ambiente seguro, uma cultura favorável à colaboração nas organizações, a qual produz ciclos (loops) de feedback positivos que se retroalimentam.

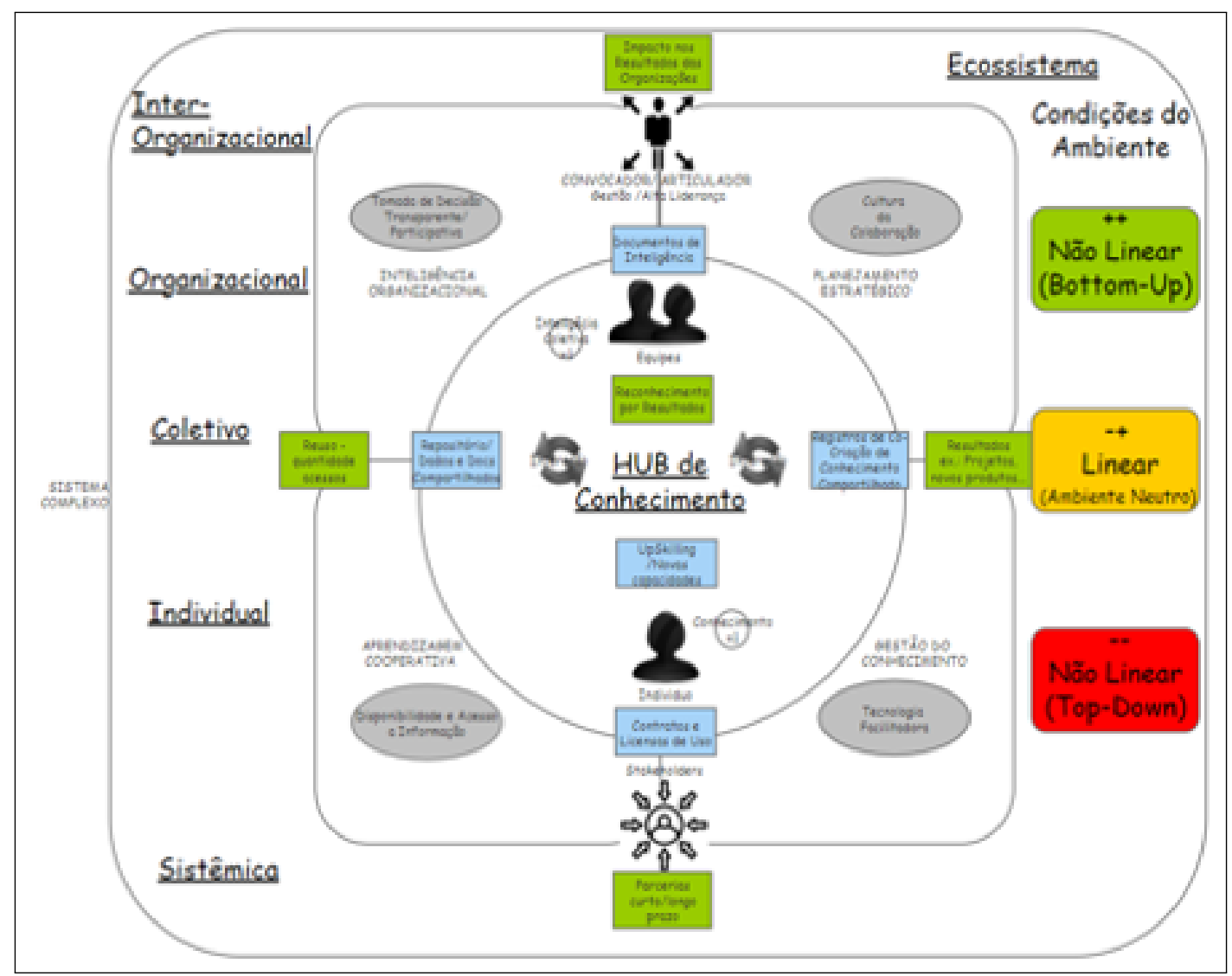

Figura 3. Framework do Modelo de Colaboração Sistêmica. Fonte: Os autores (2021).

No Framework proposto (Figura 3), identificou-se como as condições do ambiente de colaboração impactam essas sinergias do Ecossistema (System Modeling): Positivamente (Movimento Bottom-up), Neutra (Linear hora positivo e hora negativo) ou Negativamente (Movimento Top-Down). O modelo apresenta sua centralidade nas pessoas/agentes, uma vez que suas interações e o intencional compartilhamento do conhecimento são considerados fatores "sociais" da disseminação do conhecimento no ecossistema.

Assim, buscou-se identificar como a Colaboração, ao incentivar o compartilhamento do conhecimento, pode produzir resultados mensuráveis em "estoques" de conhecimento disponíveis (em azul), e produzir resultados efetivos (verde). Foram desenhados os quatro quadrantes como estruturas complementares que dão suporte ao desenvolvimento de um "Hub de Conhecimento": uma leitura do que é o estado emergente de constante evolução do centro para as extremidades, e propõe observar o movimento em escala: [Indivíduo $>$ Coletivo $>$ Organizacional $>$ Interorganizacional $>$ Sistêmica].

Pode-se sobrepor ao contexto do sistema complexo uma leitura da dinâmica deste sistema, inspirada pela: "Espiral do Conhecimento" (Nonaka, Toyama, \& Konno, 2000), que representa um processo contínuo, onde o indivíduo ascende a cada ciclo em uma nova fase de desenvolvimento de seu conhecimento (metáfora da Espiral), criando as condições para um ambiente sustentável à colaboração de longo prazo- com um potencial gerador de resultados efetivos para esse Ecossistema. 
Para compor com essa leitura, foram propostos como conceitos de base para o modelo: "Aprendizagem Cooperativa": que tem sua base na teoria da "interdependência social" entre agentes, podendo ser uma interdependência positiva ou negativa (Johnson \& Johnson, 2009).

"GC e a Criação de Conhecimento": Com os ciclos de CG conectados por meio da aprendizagem cooperativa - a cognição induz a construção do conhecimento que, por sua vez, instiga a motivação que perpetua o processo de aprendizagem. O ciclo virtuoso entre individual e coletivo, como proposto pelo modelo SECI, acrônimo de Socialização, Externalização, Combinação e Internalização (Nonaka, Takeuchi, \& Umemoto, 1996).

O ambiente, portanto, é fundamental para o desenvolvimento da colaboração e, consequentemente, a construção da inteligência coletiva. Assim, ao abordar o "planejamento estratégico" para a construção da "inteligência organizacional", observaram-se os fundamentos dos processos participativos e da administração complexa tomando como base o funcionamento dos "sistemas complexos adaptativos" pautados por: autonomia, cooperação, agregação e auto-organização (Agostinho, 2003). Por último, pode-se considerar que o sistema se organiza para produzir a transformação de dados/informação, ao incentivar o compartilhamento de conhecimento entre agentes, buscando potencializar a inteligência coletiva, base da Hierarquia da Informação (Moresi, 2000). Com o modelo, uma leitura para os resultados de GC, que, ao serem potencializados com um ambiente favorável à Colaboração, questionam o modelo Top-Down de tomada de decisões da organização e, assim, valorizam a participação dos diferentes agentes como uma solução às complexidades da gestão organizacional (Neves et al., 2018).

\section{CONCLUSÕES}

Em conclusão, ao propor esse short paper identificou-se uma oportunidade de reflexão sobre a complexidade da Colaboração, com uma possibilidade de embasamento da intersecção entre a colaboração e o compartilhamento do conhecimento, reforçada pelo contexto do ecossistema das organizações. Com as análises dos estudos levantados, foi possível produzir validações desta relação entre colaboração e o compartilhamento do conhecimento de forma visual e, a partir desta visualização, apontou-se uma lacuna na leitura do contexto da colaboração nas organizações.

Buscou-se, assim, iniciar um processo de desenvolvimento de um modelo de colaboração que representasse tanto a complexidade das relações entre agentes, quanto apontasse um potencial de compartilhamento do conhecimento nas organizações. A publicação do modelo preliminar permite aos pesquisadores propor a necessária abertura e discussões para uma constante evolução do modelo, além de apontar as possibilidades de continuação deste trabalho. Assim, espera-se simular as condições "favoráveis"e "ideais" ao ambiente de colaboração via Insight Maker. Identificou-se o potencial do estudo pela relevância e atualidade dos temas da Colaboração e do Compartilhamento do Conhecimento. E dessa forma, produzir conteúdo alinhado às demandas na área da GC, a exemplo do "KM World Conference 2021: Impacting The Future: New Models \& Tech For Knowledge Sharing"e ao Congresso do "KM Brasil 2021: Colaboração, Transformação e Inovação". 


\section{REFERÊNCIAS}

Agostinho, M. C. E. (2003). Administração complexa": revendo as bases científicas da administração. RAE Eletrônica, 2(1).

Camargo, B. V., \& Justo, A. M. (2007). Tutorial para uso do software de análise textual iramuteq. Florianópolis, SC: Universidade Federal de Santa Catarina.

Dagnino, R. (2010). Tecnologia social: ferramenta para construir outra sociedade (v. 2). São Paulo: Komedi.

Dagnino, R. (2014). Tecnologia social: contribuições conceituais e metodológicas. João Pessoa: Edupb. Recuperado de http://books.scielo.org/id/7hbdt

Johnson, D. W., \& Johnson, R. T. (2009). An educational psychology success story: Social interdependence theory and cooperative learning. Educational Researcher, 38(5), 365-379.

Kitchenham, B., \& Charters, S. (2007). Guidelines for performing systematic literature reviews in software engineering.

Moresi, E. A. D. (2000). Delineando o valor do sistema de informação de uma organização. Ciência da Informação, 29(1), 14-24.

Neves, M. L. C., Varvakis, G. J., \& Fialho, F. A. P. (2018). Pessoas, processos e tecnologia na gestão do conhecimento: uma revisão da literatura. Revista de Ciências da Administração, 20(51), 152-167.

Nobre, A. D. (2019). Selvagem ciclo de estudos sobre a vida. Recuperado de https://youtu.be/Nhom_vWVFos

Nonaka, I., Takeuchi, H., \& Umemoto, K. (1996). A theory of organizational knowledge creation. International Journal of Technology Management, 11(7-8), 833-845.

Nonaka, I., Toyama, R., \& Konno, N. (2000). Seci, ba and leadership: a unified model of dynamic knowledge creation. Long Range Planning, 33(1), 5-34.

Norman, D. A., \& Stappers, P. J. (2015). Designx: complex sociotechnical systems. She Ji: The Journal of Design, Economics, and Innovation, 1(2), 83-106.

Wood, D. J., \& Gray, B. (1991). Toward a comprehensive theory of collaboration. The Journal of Applied Behavioral Science, 27(2), 139-162.

Como citar este shortpaper (APA):

Colicchio, T. A. \& Zambon, A. C. (2021). Modelo de colaboração sistêmica: potencializando o compartilhamento do conhecimento no ecossistema das organizações. AtoZ: novas práticas em informação e conhecimento, 10(3), 1 - 7. Recuperado de: http://dx.doi.org/ 10.5380/atoz.v10i3.81279 